\title{
Semiclassical Gravity and Large-Scale Structure
}

\author{
C. Bertoni E. Carretti F. Finelli A. Messina \\ G. Venturi
}

Dipartimento di Fisica, Università degli Studi di Bologna

Istituto Nazionale di Fisica Nucleare, Sezione di Bologna

via Irnerio, 46

40126 Bologna - Italy

\begin{abstract}
The possible cosmological effects of primordial fluctuation corrections to the evolution equation of matter obtained from the Wheeler-De Witt equation are explored. In particular, both the metric and a scalar matter field are expanded around their homogeneous values and the corrections induced on the scalar field fluctuation spectrum are perturbatively estimated. Finally, results of a preliminary numerical simulation to investigate the effects on large-scale structure formation are presented.
\end{abstract}

The matter-gravity system may be studied quantum-mechanically through the canonical quantization of gravity within the superspace approach [1] [2]. One then obtains the Wheeler-De Witt (WD) equation and a matter-gravity wave function which, in analogy with the Born-Oppenheimer (BO) studies of molecules [3], may be factorized into two parts: one involving only gravitational degrees of freedom and the other involving both the gravitational and the matter degrees of freedom. Correspondingly, the WD equation in which time is absent is split into two pieces: one describing gravitation in an effective potential given by the mean energy-momentum tensor of matter and the other describing the matter whose evolution is parametrized by time which is derived from the semiclassical approximation to the gravitational wave function [4] [5]. The above is contingent on the hypothesis that the Planck mass is much larger than the mass of any matter field or any inverse length scale used to describe matter.

The above approach has been examined in detail within the context of a minisuperspace model with matter [6] with the view of understanding under what conditions the BO approximation is valid and quantum cosmology leads to the usual physics (Schrödinger equation for matter and Einstein classical equations for gravity on scales larger than the Planckian). It was found that such was the case in an inflationary scenario [8] and after ten or more Planck times the 
usual physics ensues. Such a result was obtained by estimating perturbatively the fluctuation corrections due to quantum gravitational effects both on the equation of motion for the purely gravitational part and that for matter, since the actual solution of the coupled nonlinear equations is extremely complicated.

The fluctuation corrections considered were a consequence of matter-gravity forming a closed system: fluctuations of gravitational origin generate corresponding fluctuations in the evolution of matter and it is such fluctuations that are neglected in the BO approximation. Henceforth we shall refer to them as fluctuation corrections.

It has been previously suggested that inflation could in principle provide a causal mechanism for the origin of density perturbations that later grow to form large-scale structures [9], thus we feel it is of interest to explore the influence of the above fluctuation corrections in such a context. In order to do so we generalize the previous analysis [6] to include perturbations both for the threemetric and for the scalar field associated with inflation. In particular, both the perturbations of the three metric and the scalar field (which represents matter and whose non-zero vacuum expectation value has been incorporated in the cosmological constant) are expanded in terms of scalar harmonics on the three sphere and just retained to second order [10]. We then consider the resulting coupled equations in the presence of a positive cosmological constant (de Sitter) with the scope of obtaining information on the large-scale structures.

Since we can not actually solve the nonlinear coupled matter-gravity system we do not know the effect of fluctuation corrections on the scalar field energy and the resulting distorted scalar field spectrum. However we can estimate the fluctuation corrections perturbatively using, as unperturbed solution, the matter wave function obtained on neglecting them (BO approximation). One then assumes that the distorted scalar field spectrum is reflected by the relative weight of the modes (unperturbed plus contributions due to fluctuation corrections) at a given instant, say when each mode exits the horizon during the inflationary era [12]. It is further clear that the relative strength of the diverse corrections besides depending on the moment at which they are frozen will also depend on the initial value of the cosmological constant, in any case the fluctuation corrections must always be less than the unperturbed result otherwise our perturbative approach is meaningless.

In order to illustrate our approach we recall the perturbed minisuperspace results previously obtained [10]. The spatial metric $h_{\alpha \beta}$ is given by

$$
h_{\alpha \beta}=a^{2}(\eta)\left(\Omega_{\alpha \beta}+\epsilon_{\alpha \beta}\right),
$$

where $a$ is the Robertson-Walker scale factor, $\eta$ the conformal time, $\Omega_{\alpha \beta}$ the metric on a sphere of unit radius and $\epsilon_{\alpha \beta}$ a perturbation to it which can be expanded in terms of the spherical harmonics of $S^{3}$ [10]: 


$$
\epsilon_{\alpha \beta}=\sum_{n, l, m}\left[6^{\frac{1}{2}} a_{n l m} \frac{1}{3} \Omega_{\alpha \beta} Q_{l m}^{n}+6^{\frac{1}{2}} b_{n l m}\left(P_{\alpha \beta}\right)_{l m}^{n}\right],
$$

where the coefficients $a_{n l m}, b_{n l m}$ are functions of $\eta$ but not of the spatial coordinates. Similarly, one may also expand the lapse $(N)$, shift $\left(N_{\alpha}\right)$ functions and the scalar (matter) field $\Phi$ :

$$
\begin{gathered}
N=a\left[1+6^{-\frac{1}{2}} \sum_{n, l, m} g_{n l m} Q_{l m}^{n}\right], \\
N_{\alpha}=a \sum_{n, l, m} 6^{-\frac{1}{2}} k_{n l m}\left(P_{\alpha}\right)_{l m}^{n} \\
\Phi=\phi(\eta)+\sum_{n, l, m} f_{n l m} Q_{l m}^{n} .
\end{gathered}
$$

and we have just considered terms in the expansions eqs. (2)-(5) associated with scalar harmonics $Q_{l m}^{n}$ on the three sphere (or their covariant derivatives [11) since it is these contributions which are relevant for the non-homogeneous part of the scalar field in the semiclassical limit 10]. Henceforth, for the sake of brevity, we shall only exhibit the index $n$, other indices $(l, m)$ being understood.

One may now substitute the above into the total Hamiltonian density (gravitation plus matter) and perform the spatial integration keeping the minisuperspace variable $a$ to all orders and the perturbations $\phi, a_{n}, b_{n}, f_{n}, k_{n}$ only to second order. These now become the dynamical variables and one may now quantize canonically obtaining the following WD equation and momentum conditions:

$$
\begin{gathered}
\left(\hat{H}_{\mid 0}+\sum_{n}{ }^{S} \hat{H}_{\mid 2}^{n}\right) \Psi \equiv\left(\hat{H}^{G}+\hat{H}^{M}\right) \Psi=0, \\
\hat{H}_{\mid 1}^{n} \Psi={ }^{S} \hat{H}_{-1}^{n} \Psi=0 .
\end{gathered}
$$

The indices 0,1 and 2 indicate the order with respect to the perturbations, and $S$ the scalar part of the total Hamiltonian (obtained by setting to 0 the vector and tensor perturbations in $[10]$ ). $\hat{H}^{G}$ and $\hat{H}^{M}$ are respectively the gravitational and matter parts of the Hamiltonian and $\Psi\left(a,\left\{a_{n}\right\},\left\{b_{n}\right\}, \phi,\left\{f_{n}\right\}\right)$ is the total matter-gravity wave function.

We now follow a procedure analogous to the one illustrated elsewhere [6] [7] and factorize the wave function $\Psi$ as: 


$$
\begin{aligned}
\Psi\left(a,\left\{a_{n}\right\},\left\{b_{n}\right\}, \phi,\left\{f_{n}\right\}\right) & =\prod_{n} \tilde{\chi}_{n}\left(a, a_{n}, b_{n}, \phi, f_{n}\right) \tilde{\psi}_{n}\left(a, a_{n}, b_{n}\right) \\
& =\prod_{n} \tilde{\chi}_{n} \prod_{l} \tilde{\psi}_{l} \equiv \tilde{\chi} \tilde{\psi}
\end{aligned}
$$

which on substituting into eq. (6) leads to the following coupled equations:

$$
\begin{gathered}
\left(\hat{H}^{G}+\left\langle\hat{H}^{M}\right\rangle\right) \tilde{\psi}=-\left\langle\hat{H}_{k i n}^{G}\right\rangle \tilde{\psi} \\
\left(\hat{H}^{M}-\left\langle\hat{H}^{M}\right\rangle\right) \tilde{\chi}+\frac{\hbar^{2}}{m_{p}^{2}}(\nabla \log \tilde{\psi})^{T} G \nabla \tilde{\chi}=-\left(\hat{H}_{k i n}^{G}-\left\langle\hat{H}_{k i n}^{G}\right\rangle\right) \tilde{\chi},
\end{gathered}
$$

where $\hat{H}_{\text {kin }}^{G}$ is the gravitational kinetic energy term and the averaging procedure \langle\rangle is over all matter field configurations. Further $G$ is a matrix depending on the diverse gravitational degrees of freedom, $\nabla$ is a vector gradient with respect to the various gravitational degrees of freedom and $\nabla^{T}$ its transpose. It is understood that eq. (10) is evaluated where $|\tilde{\psi}|^{2}$ has support [7]. The terms on the r.h.s. of eqs. (9) and (10) are associated with fluctuations and disappear in the $\mathrm{BO}$ approximation. Analogous equations are obtained from the constraints eq. (7).

We may now introduce a semiclassical approximation to the gravitational wave function through:

$$
\tilde{\psi}\left(\tilde{a},\left\{a_{n}+b_{n}\right\}\right)=N_{G} e^{\frac{i}{\hbar} S^{G}},
$$

with

$$
\tilde{a}=a e^{\frac{1}{2} \sum_{n} a_{n}^{2}-2 \sum_{n} \frac{n^{2}-4}{n^{2}-1} b_{n}^{2}},
$$

where $S^{G}$ is the classical gravitational action and is solution to the HamiltonJacobi equation obtained for $\hbar \rightarrow 0$ from eq. (9) in the absence of fluctuations, and the contribution to the lower order (in $\hbar$ ) from $N_{G}$ is negligible in our present approximation. Further, since the momentum constraints eq. (7) are associated with reparametrisation invariance and in general reduce the number of parameters leading to a mixing of matter and gravitation degrees of freedom 122 in contrast with our factorization ansatz eq. (8), we require that they be satisfied just for the gravitational wave function $\psi$ in the absence of matter backreaction [12]. This then leads to the above parameter dependence in $\tilde{\psi}$.

Through the above, as previously explained [4]-[6], one may introduce a conformal time $\eta$ and eq. (10) becomes: 


$$
\begin{aligned}
\left(\hat{H}^{M}-\left\langle\hat{H}^{M}\right\rangle\right) \tilde{\chi}-\frac{i \hbar}{m_{p}^{2}}\left(\nabla \log S^{G}\right)^{T} G \nabla \tilde{\chi} & =\left(\hat{H}^{M}-\left\langle\hat{H}^{M}\right\rangle\right) \tilde{\chi}-i \hbar \frac{\partial}{\partial \eta} \tilde{\chi} \\
& =-\left(\hat{H}_{\text {kin }}^{G}-\left\langle\hat{H}_{\text {kin }}^{G}\right\rangle\right) \tilde{\chi}
\end{aligned}
$$

and in particular on neglecting the fluctuation in eq. (13) one obtains:

$$
\left(\hat{H}^{M}-i \hbar \frac{\partial}{\partial \eta}\right) e^{-\frac{i}{\hbar} \int{ }^{\eta}\left\langle\hat{H}^{M}\right\rangle d \eta^{\prime}} \tilde{\chi} \equiv\left(\hat{H}^{M}-i \hbar \frac{\partial}{\partial \eta}\right) \chi_{s}=0,
$$

which is the usual evolution equation for matter. Eq. (14) is then solved through an ansatz for $\chi_{s}$ 13:

$$
\chi_{s}=N e^{\frac{i}{h} S}
$$

where:

$$
\begin{aligned}
S= & S_{0}(a, \phi)+ \\
& \sum_{n}\left(\frac{1}{2} S_{a a}^{(n)} a_{n}^{2}+\frac{1}{2} S_{b b}^{(n)} b_{n}^{2}+\frac{1}{2} S_{f f}^{(n)} f_{n}^{2}+\right. \\
& \left.S_{a b}^{(n)} a_{n} b_{n}+S_{a f}^{(n)} a_{n} f_{n}+S_{b f}^{(n)} b_{n} f_{n}\right),
\end{aligned}
$$

which is substituted into eq. (14). On equating coefficients of the same order in $a_{n}, b_{n}, f_{n}$, a series of relations are then obtained and solved for the functions $S^{(n)}(a, \phi)$.

The solutions for $\tilde{\psi}$ and $\tilde{\chi}$ are then used to obtain expressions of physical interest. Further, since a semiclassical limit for $\tilde{\psi}$ is considered, for the values of $a, a_{n}$ and $b_{n}$ one uses their average (classical) values. In particular, from the matter wave function $\chi_{s}$ (solution to eq. (14)) one determines the expectation values of the coefficients $f_{n}^{2}$ at the exit from the horizon, obtaining with $H$ the Hubble parameter:

$$
\left\langle f_{n}^{2}\right\rangle \simeq \frac{\hbar H^{2}}{2 n^{3}}
$$

which is directly related to the spectrum of density fluctuations and corresponds to the Harrison-Zel'dovich (HZ) spectrum (since $k=\frac{n}{2 \pi a}$, with $k$ the physical wave number) [14]. Further, one may estimate, using the lowest order solution of eq. (14), the magnitude of the fluctuations in eq. (13) through:

$$
\left(\hat{H}_{k i n}^{G}-\left\langle\hat{H}_{k i n}^{G}\right\rangle\right) \tilde{\chi} \simeq \pm\left[\left\langle\overleftarrow{\hat{H}}_{k i n}^{G} \hat{H}_{k i n}^{G}\right\rangle-\left\langle\hat{H}_{k i n}^{G}\right\rangle^{2}\right]^{\frac{1}{2}} \tilde{\chi}
$$


Figure 1: Unperturbed spectrum (HZ) and its distorsion (fluctuation corrections $+/-$ ) power spectrum at $\simeq 10^{17} \mathrm{GeV}$ and $\simeq 10^{14} \mathrm{GeV}$ inflation energy.

which may be interpreted as a correction to the scalar field energies and consequently as a distortion of the unperturbed (HZ) spectrum and we shall denote it by $\Delta\left\langle f_{n}^{2}\right\rangle$. One then obtains:

$$
\frac{\Delta\left\langle f_{n}^{2}\right\rangle}{\left\langle f_{n}^{2}\right\rangle}= \pm \frac{\left[\left\langle\overleftarrow{\hat{H}}_{k i n}^{G} \hat{H}_{k i n}^{G}\right\rangle-\left\langle\hat{H}_{k i n}^{G}\right\rangle^{2}\right]^{\frac{1}{2}}}{\left\langle\hat{H}^{M}\right\rangle}
$$

where the scale factor is evaluated at the exit from the horizon for each mode.

In particular, if the spectrum is evaluated at a common time after having re-entered the horizon, one finds that the unperturbed part behaves as $n$, while the corrections behave as $\simeq n^{-1}$ and $\simeq n^{2}$ for small and large $n$ respectively and are therefore most effective both at extremely large and small scales. Further the interval in $n$ for which our perturbative approach is valid increases as the cosmological constant driving inflation is decreased (see Fig. 1). For example, for an inflation energy $\simeq 10^{17} \mathrm{GeV}$ and an $\mathrm{e}$-folding of 100 , the $\mathrm{HZ}$ spectrum is modified by our perturbation on physical scales in the interval $\left(1 \div 10^{4}\right) h^{-1} M p c$ (we adopt the value $h=0.5$ for the present value of the Hubble constant $H_{0}$ in units of $100 \mathrm{~km} \mathrm{~s}^{-1} \mathrm{Mpc}^{-1}$ ). However, from the analysis of present inflationary models 15] one obtains that standard and chaotic inflation is consistent with the COBE results [16] [17] only for energies $\simeq 10^{14} \mathrm{GeV}$ (which is the energy scale for which the observable fluctuations exit the horizon) and in order to obtain a higher energy one should construct models with suitable e-folding and/or a horizon growth during inflation steeper than that presently used [15].

With the fluctuation spectra obtained ( $\mathrm{HZ} \pm$ fluctuation corrections) and for an inflation energy of $\simeq 10^{14} \mathrm{GeV}$, we have performed numerical simulations assuming cold dark matter dominance (the so-called CDM model): the constituents of dark matter in this model are massive particles, which decoupled from radiation when non-relativistic or never were in thermal equilibrium.

In the last decade the standard CDM model has shown a high predictive power in explaining many observed properties of the large-scale galaxy distribution. However, it is now known that this model has some serious problems, mostly due to the high ratio of small to large-scale power. In particular, the COBE normalization [16] [17] implies excessive velocity dispersion on $M p c$ scales [18] and is unable to reproduce the slope of the galaxy angular correlation function obtained from the APM survey [19]. The spectrum of the primordial fluctuations in the CDM model is the $\mathrm{HZ}$ spectrum and, as mentioned, our solutions modify this spectrum at small and very large scales.

To follow the non-linear evolution after the matter-radiation decoupling we used a particle-mesh code [20]. A preliminary analysis with $N_{p}=128^{3}$ 
Table 1: Comparison of observed and computed mass variance.

$\begin{array}{cccc}h^{-1} M p c & A P M[21 & Q F_{-} & H Z \\ 10 & 1.05-1.47 & 1.10-2.08 & 2.31-3.07 \\ 15 & 0.61-0.89 & 0.58-0.85 & 0.99-1.19 \\ 20 & 0.39-0.62 & 0.35-0.43 & 0.55-0.57 \\ 25 & 0.29-0.49 & 0.21-0.24 & 0.31-0.33 \\ 30 & 0.18-0.33 & 0.13-0.16 & 0.18-0.21\end{array}$

particles and $N_{g}=128^{3}$ grid-points indicated that adding or subtracting our fluctuation corrections to the HZ spectrum in the large $n$ region, increased or decreased respectively the mass excess or the bulk velocities with respect to the HZ results. Therefore, we proceeded with more detailed simulations with $N_{p}=256^{3}$ particles on $N_{g}=256^{3}$ grid-points, on a Cray T3D MCA 64-8.

We ran three simulations, one with the HZ spectrum over a box of size $128 h^{-1} M p c$ and two with the spectrum obtained on subtracting the fluctuation corrections from the $\mathrm{HZ}$ spectrum (we shall denote it by $Q F_{-}$), one over a box of size $128 h^{-1} M p c$ and the other over a box of size $512 h^{-1} M p c$.

The amplitude of the primordial fluctuation spectrum is not determined by our free theoretical parameters (that is, the inflation energy and the efolding number) but by the inverse of the rms mass fluctuation on a sharpedged sphere of radius $8 h^{-1} M p c\left(\sigma_{8}\right)$. The COBE DMR detection of large angular scale anisotropies of the cosmic microwave background [16] then fixes the normalization and makes the model completely specified.

A detailed study of the numerical results is under way, however a preliminary analysis of the variance of mass reported in Table 1 for the box of size $128 h^{-1} M p c$ (the range of values for $Q F_{-}$and HZ refers to the greatest and the least of three evaluations obtained following [21]) gives an idea of the relevance of the fluctuation corrections to the development of large scale structure. Analysis of mock galaxy catalogues obtained by such simulations should then provide a quantitative answer concerning the possibility of solving the two major problems of the CDM model with quantum corrections.

Further details of theoretical and numerical aspects will be presented elsewhere 22] 23].

\section{References}

[1] J. A. Wheeler, in Relativity, Groups and Topology (Les Houches Lectures, 1963), ed. C. de Witt and B. de Witt, New York (Gordon and Breach)

[2] B. S. De Witt, Phys. Rev., 160, 1113 (1967)

[3] For a discussion of the Born-Oppenheimer (or adiabatic in the quantum mechanical sense) approximation see for example A. Messiah, Quantum 
Mechanics, vol. 2, Wiley, New York (1962) or L. I. Schiff, Quantum Mechanics, McGraw-Hill, New York (1955).

[4] T. Banks, Nucl. Phys. B, 249, 332 (1985)

[5] R. Brout, Found. Phys. 17, 603 (1987)

[6] R. Brout, G. Venturi, Phys. Rev. D, 39, 2436 (1989)

[7] G. Venturi, in Differential Geometric Methods in Theoretical Physics, eds. L.-L. Chau W. Nahm, p. 703, Plenum, New York (1990)

[8] R. Brout, F. Englert, E. Gunzig Gen. Rel. Grav., 10, 1 (1979)

A. H. Guth, Phys. Rev. D, 23, 347 (1981)

[9] A. H. Guth, S.-Y. Pi, Phys. Rev. Lett., 41, 1110 (1982)

A. A. Starobinsky, Phys. Lett. B, 117, 178 (1981)

S. W. Hawking, Phys. Lett. B, 117, 295 (1981)

T. M. Bardeen, P. J. Steinhardt, M. J. Turner, Phys. Rev. D, 26, 679 (1981)

[10] J. J. Halliwell, S. W. Hawking, Phys. Rev. D, 31, 1777 (1985), we essentially employ the same notation as in this reference.

[11] In particular $P_{\alpha \beta}=\frac{1}{n^{2}-1} Q_{\mid \alpha \beta}+\frac{1}{3} \Omega_{\alpha \beta} Q$.

[12] S. Wada, Nucl. Phys., B, 276, 729 (1986)

I. Shirai, S. Wada, Nucl. Phys., B, 303, 728 (1988)

[13] See for example J. Guven, B. Lieberman, C. T. Hill, Phys. Rev. D, 39, 438 (1989)

[14] P. J. E. Peebles, The large scale structure of the Universe, Princeton University Press, Princeton (1980)

[15] M. S. Turner, Phys. Rev. D, 48, 3502 (1993)

M. S. Turner, Phys. Rev. D, 48, 5539 (1993)

[16] G. F. Smoot, et al, ApJ, 396, 396 (1992)

[17] C. L. Bennet, et al, ApJ, 436, 423 (1994)

[18] J. M. Gelb, E. Bertschinger, ApJ, 436, 491 (1994)

[19] S. J. Maddox, G. Efstathiou, W. J. Sutherland, J. Loveday, Mon. Not. R. Astron. Soc., 242, 43P (1990)

[20] A. Messina, F. Lucchin, S. Matarrese, L. Moscardini, Astropar. J., 1, 99 (1992) 
[21] J. Loveday, G. Efstathiou, B. A. Peterson, S. J. Maddox, ApJ, 400, L43 (1992)

[22] C. Bertoni, Ph.D. Thesis, University of Bologna (1995)

[23] C. Bertoni, E. Carretti, F. Finelli, A. Messina, G. Venturi, "The Semiclassical Limit of Quantum Gravity and the Large-Scale Structure of the Universe", in preparation (1995) 